\title{
Personality Disorders Psychiatric and Psychotherapeutic Practices
}

\author{
Dr. Meghamala. S. Tavaragi ${ }^{1}$, Mrs. Sushma. $C^{2}$, Mr. Susheelkumar V. Ronad ${ }^{3}$
}

\section{ABSTRACT:}

Personality disorder is a common and chronic disorder. Its prevalence is estimated between 10 and 20 percent in the general population, this means that at least one in every five to ten individuals in the community has personality disorder. In general, personality disorders are diagnosed in 40-60 percent of psychiatric patients, making them the most frequent of all psychiatric diagnoses. Personality disorders are a class of mental disorders characterized by enduring maladaptive patterns of behavior, cognition and inner experience, exhibited across many contexts and deviating markedly from those accepted by the individual's culture.

There are many different forms (modalities) of treatment used for personality disorders: Individual psychotherapy has been a mainstay of treatment. There are long-term and short-term (brief) forms; Family therapy including couples therapy; Group therapy for personality dysfunction is probably the second most used; Psychological-education may be used as an addition; Self-help groups may provide resources for personality disorders; Psychiatric medications for treating symptoms of personality dysfunction or co-occurring conditions; Milieu therapy, a kind of group-based residential approach, has a history of use in treating personality disorders, including therapeutic communities.

Keywords: Personality disorder, Prevalence, Long and short term psychotherapeutic intervention.

Personality disorders are a class of mental disorders characterized by enduring maladaptive patterns of behavior, cognition and inner experience, exhibited across many contexts and deviating markedly from those accepted by the individual's culture. These patterns develop early, are inflexible and are associated with significant distress or disability (American Psychological Association, 2006). -

${ }^{1}$ Psychiatrist Dept of Psychiatry, Dimhans Dharwad; Karnataka

${ }^{2}$ Clinical Psychologist, Dept of Psychology, Dimhans Dharwad; Karnataka

${ }^{3}$ Assistant Professor Dept of Psychiatric Nursing, DIMHANS Dharwad Karnataka

(C) 2015 I M Tavaragi, C Sushma, S Ronad; licensee IJIP. This is an Open Access Research distributed under the terms of the Creative Commons Attribution License (http://creativecommons.org/licenses/by/2.0), which permits unrestricted use, distribution, and reproduction in any Medium, provided the original work is properly cited. 
-Personality disorders are defined by experiences and behaviors that differ from societal norms and expectations. Those diagnosed with a personality disorder may experience difficulties in cognition, emotiveness, interpersonal functioning or control of impulses. Personality disorder is a common and chronic disorder. Its prevalence is estimated between 10 and 20 percent in the general population, this means that at least one in every five to ten individuals in the community has personality disorder. In general, personality disorders are diagnosed in 40-60 percent of psychiatric patients, making them the most frequent of all psychiatric diagnoses [Sab, 2001]. The increased clinical recognition of personality disorder probably reflects its increased presence in the general population. Personality disorder is also a predisposing factor for other psychiatric disorders, possible explanation for the increased interest in personality disorder is its position somewhere in between minor and major psychiatric problems (e.g., adjustment disorder vs. schizophrenia), which makes it interesting to a wide variety of experts and schools, ranging from purely sociodynamic to purely biological. There are numerous possible causes of mental disorders. Genetic dispositions as well as particular life experiences, which may or may not include particular incidents of trauma or abuse. Child abuse and neglect consistently evidence themselves as antecedent risks to the development of personality disorders in adulthood [Cohen, Brown, \& Smailes, 2001].

Accordingly, diagnosis and treatment of any psychiatric patient, as well as patients with psychosocial problems, and prevention planning in many medical syndromes are inadequate without a systematic approach to assessing and classifying personality. Finally, the development of psychiatry as a whole has generated an increased interest in personality disorder.

\section{CLASSIFICTION OF PERSONALITY DISORDERS}

The leading classifications of personality disorder are the International Classification of Diseases (ICD) of the World Health Organization and the DSM of the American Psychiatric Association. DSM-IV-TR defines personality disorders as an enduring pattern of inner experience and behavior that deviates markedly from the expectations of the individual's culture. The pattern is manifested in two (or more) of the following areas:

- cognition (i.e., ways of perceiving and interpreting self, other people, and events)

- affectivity (i.e., the range, intensity, liability and appropriateness of emotional response)

- interpersonal functioning

- impulse control

The pattern is stable and of long duration and its onset can be traced back at least to adolescence or early adulthood. It is inflexible and pervasive across a broad range of personal and social situations and leads to clinically significant distress or impairment in social, occupational, or other important areas of functioning.

Personality disorder subtypes classified in DSM-IV-TR are: schizotypal, schizoid, and paranoid (Cluster A); narcissistic, borderline, antisocial, and histrionic (Cluster B); and obsessivecompulsive, dependent, and avoidant (Cluster C). 
The ICD-10 section on mental and behavioral disorders includes categories of personality disorder and enduring personality changes. They are defined as ingrained patterns indicated by inflexible and disabling responses that significantly differ from how the average person in the culture perceives, thinks and feels, particularly in relating to others [WHO, 2010].

The specific personality disorders are: paranoid, schizoid, dissocial, and emotionally unstable (borderline type and impulsive type), histrionic, anankastic, anxious (avoidant) and dependent [ICD-10, 2010].

\section{1)PARANOID PERSONALITY DISORDER:}

The hallmarks of paranoid personality disorder are excessive suspiciousness and distrust of others expressed as a pervasive tendency to interpret actions of others as deliberately demeaning, malevolent, threatening, exploiting, or deceiving.

Incidence and prevalence: Prevalence rates of 0.5 to 2.5 percent in the general population, 10 to 30 percent for psychiatric inpatients, and 2 to 10 percent for psychiatric outpatients are reported in DSM-IV-TR. this disorder is more commonly diagnosed in men.

Famlial pattern and genetics: Some studies have demonstrated increased prevalence of this personality disorder among relatives of probands with chronic schizophrenia and delusional disorder, paranoid type.

Causes - Specific psychopathology: Increased use of projection, lack basic self confidence

Co morbidity: (OCD), agoraphobia, and substance abuse or dependence. The most common cooccurring personality disorders These patients are at increased risk for major depression, obsessive-compulsive disorder are schizotypal, schizoid, narcissistic, avoidant, and borderline.

Paranoid personality disorder has been postulated to be a premorbid antecedent of delusional disorder, paranoid type.

Impairment: Impairment is frequently only mild and typically includes occupational and social difficulties

Complications: Complications include brief reactive psychosis, particularly in response to stress.

Intervention strategies: Psychotherapy is the treatment of choice. Therapists should be straightforward in dealings with these patients. Clinicians' overzealous use of interpretationespecially interpretation about deep feelings of dependence, sexual concerns, and wishes for intimacy-increase patients' mistrust significantly. Paranoid patients usually do not do well in group psychotherapy, Delusional accusations must be dealt with realistically but gently and without humiliating patients. 


\section{2) SCHIZOID PERSONALITY DISORDER:}

The hallmarks of schizoid personality disorder are a pervasive pattern of social detachment and a restricted range of expressed emotions in interpersonal settings beginning by early adulthood

Incidence and prevalence: Prevalence rates varying from uncommon (DSM-IV-TR) to 7.5 percent in the general population. According to DSM-IV-TR, this disorder is more commonly diagnosed in men and may cause more impairment in them.

Famlial pattern and genetics: An increased prevalence among the relatives of probands with schizophrenia or schizotypal personality disorder has been reported.

Causes - Specific pychopathology: Increased use of withdrawal, increased fantasy

Comorbidity: This personality disorder sometimes appears as the premorbid antecedent of delusional disorder, schizophrenia, or, rarely, major depression. The most common cooccurring personality disorders are paranoid, schizotypal, and avoidant

Impairment: Impairment includes frequently severe problems in social relations. Occupational problems develop when interpersonal involvement is required; solitary work sometimes favorably affects overall performance

Complications: Complications include very brief reactive psychosis, particularly in response to stress.

Intervention stratergies: The treatment of patients with schizoid personality disorder is similar to that of those with paranoid personality disorder.. As trust develops, schizoid patients may, with great trepidation, reveal a plethora of fantasies, imaginary friends, and fears of unbearable dependence — even of merging with the therapist.

In group therapy settings, patients with schizoid personality disorder may be silent for long periods. The patients should be protected against aggressive attack by group members.

\section{3) SCHIZOTYPAL PERSONALITY DISORDER:}

Schizotypal personality disorder is characterized by social and interpersonal deficits as indicated by pervasive discomfort with reduced capacity for close relationships, as well as cognitive and perceptual distortions and eccentric behavior (not severe enough to meet criteria for schizophrenia).

Incidence and prevalence: A prevalence rate of 3 percent in the general population is reported in DSM-IV-TR. earlier reports suggested a range between 2 and 6 percent. The sex ratio is unknown; this disorder is frequently diagnosed in women with fragile $\mathrm{X}$ syndrome.

Familial pattern and genetics: There is an increased prevalence of this personality disorder among the first-degree relatives of probands with schizophrenia. Also, there is an increased prevalence of schizophrenia and other psychoses in the relatives of probands with schizotypal personality disorder. The disorder itself tends to aggregate in families (DSM-IV-TR). 
Causes - Specific psychopathology: Close to schizoid spectrum disorder.

Comorbidity: More than one-half of these patients have had at least one episode of major depression, and 30 to 50 percent have major depression concurrent with this personality disorder. The most common cooccurring personality disorders are schizoid, paranoid, avoidant, and borderline.

Impairment: Impairment typically includes occupational and social difficulties.

Complications: Complications include transient psychotic episodes, particularly in response to stress. Symptoms sometimes become so significant that they meet criteria for schizophreniform disorder, delusional disorder, and brief psychotic disorder.

Intervention strategies: clinicians must deal sensitively with the patient .These patients have peculiar patterns of thinking, and some are involved in cults, strange religious practices, and the occult. Therapist must not ridicule such activities or be judgmental about these beliefs or activities.

\section{Cluster B Personality Disorders}

\section{4) ANTISOCIAL PERSONALITY DISORDER:}

The hallmarks of antisocial personality disorder are pervasive disregard for and violation of rights of others occurring since 15 years of age and continuing into adulthood. A person has to be 18 years of age or older, and there has to be evidence of conduct disorder before 15 years of age (conduct disorder involves a repetitive and persistent pattern of behavior in which the basic rights of others or major age-appropriate social rules are violated; the examples include aggression to people or animals, or both; destruction of property; deceitfulness or theft; and serious violation of rules).

Incidence and prevalence: Prevalence rates of 3 percent for men and 1 percent for women in the general population and 3 to 30 percent in clinical settings, with even higher rates for forensic samples and substance abusers, have been reported. A high frequency of antisocial personality disorder is associated with low socioeconomic status and urban settings. According to DSM-IVTR, this disorder is more commonly (by a ratio of 3 to 1 ) diagnosed in men.

Famlial pattern and genetics: Antisocial personality disorder is more frequent among the firstdegree biological relatives of probands with this disorder. Biological relatives of women with antisocial personality disorder are at increased risk for the same disorder compared to biological relatives of men with antisocial personality disorder. Genetic studies have suggested familial transmission of antisocial personality disorder, substance use, and somatization disorder, the former two being characteristic of men, and the latter being characteristic of women in the same family. Adoption studies have shown that genetic and environmental factors contribute to the risk for this disorder. Adopted and biological children of parents with antisocial personality disorder are at increased risk for this disorder. 
Causes - Specific pychopathology: social disintegration, acting out, absence of guilt, manipulative, poor superego, and novelty increased seeking

Comorbidity: These patients are at increased risk for impulse control disorders, major depression, substance abuse or dependence, pathological gambling, anxiety disorders, and somatization disorder. The most common cooccurring personality disorders are narcissistic, borderline, and histrionic

Impairment: Impairment is extremely variable and typically includes social difficulties

Course: After 30 years of age, the most flagrant antisocial behaviors (promiscuity and crime) and the less severe behaviors and substance use tend to decrease.

Complications: Complications include dysphoria, tension, low tolerance for boredom, depressed mood, and premature, violent death.

\section{5) NARCISSISTIC PERSONALITY DISORDER:}

The hallmarks of narcissistic personality disorder are a pervasive sense of grandiosity (in fantasy or in behavior), a need for admiration, a lack of empathy, and chronic, intense envy

Incidence and prevalence: Prevalence rates of 2 to 16 percent in the clinical population and less than 1 percent in the general population are reported in DSM-IV-TR. According to DSM-IV-TR, this disorder is more commonly diagnosed in men (50 to 75 percent of diagnosed cases are men).

Familial pattern and genetics: There may be a higher risk for this personality disorder in the offspring of narcissistic parents who impart on their children an unrealistic sense of grandiosity. In addition, most narcissistic persons are realistically talented, beautiful, or highly intelligent, as these features serve as the nucleus around which the sense of specialness is further organized.

Causes - Specific pychopathology: Excess investment of libidinal energy in 'self' because of severe frustration with early object .Pathological grandiose self which hides a diffuse and aimless inner identity, self hatred lies at the core rather than self lone

Comorbidity: These patients are at increased risk for major depression and substance abuse or dependence (especially cocaine use). The most common cooccurring personality disorders are borderline, antisocial, histrionic, and paranoid.

Impairment: Impairment is frequently severe and typically includes marital problems and interpersonal relationships in general.

Course: The course is chronic. However, narcissistic symptoms tend to diminish after 40 years of age, when pessimism usually develops.

Complications: Complications include social withdrawal, depressed mood, dysthymic or major depressive disorder in reaction to criticism or failure. 
Intervention strategies: Because patients must renounce their narcissism to make progress, the treatment narcissistic personality disorder is difficult. Psychiatrists such as kernberg and Heinz Kohut have advocated using psychoanalytic approaches to effect change, but much research is required to validate the diagnosis and to determine the best treatment. Some clinicians advocate group therapy for their patients so they can learn how to share with others and, under ideal circumstances can develop an empathic response to others.

\section{6) HISTRIONIC PERSONALITY DISORDER:}

The hallmarks of histrionic personality disorder are pervasive and excessive self-dramatization, excessive emotionality, and attention seeking.

Incidence and prevalence: Prevalence rates of 2 to 3 percent in the general population and 10 to 15 percent for psychiatric inpatients and outpatients are reported in DSM-IV-TR. There seems to be a general agreement that this disorder occurs far more frequently among women. According to DSM-IV-TR, the disorder might be equally frequent among men and women.

Familial pattern and genetics: This disorder tends to run in families. A genetic link between histrionic and antisocial personality disorder and alcoholism has been suggested.

Causes - Specific pychopathology: Abnormal intense attachment to parental figures, acting out, Dissociate defenses, repression, high emotionality, dramatization, exhibitionism, ego centricity, sexual provacativeness, reward dependence

Comorbidity: These patients are at increased risk for major depression, somatization disorder, and conversion disorder. The most common co-occurring disorders are narcissistic, borderline, antisocial, and dependent.

Impairment: Impairment is frequently only mild and typically includes personal romantic relationships.

Complications: Complications include frequent suicidal gestures and threats to coerce better care giving. Interpersonal relations are unstable, shallow, and generally ungratifying. There are frequent marital problems secondary to the tendency to neglect long-term relationships for the excitement of new relationships.

Intervention strategies: Patients with histrionic personality disorder are often unaware of their own real feelings; clarification of their inner feelings is an important therapeutic process. Psychoanalytically oriented psychotherapy, is probably the treatment of choice for histrionic personality disorder.

\section{7) BORDERLINE PERSONALITY DISORDER:}

The hallmarks of borderline personality disorder are pervasive and excessive instability of affects, self-image, and interpersonal relationships, as well as marked impulsivity.

Incidence and prevalence: Prevalence rates of 2 percent in the general population, 10 percent for psychiatric outpatients, 20 percent for psychiatric inpatients, and 30 to 60 percent among 
patients with personality disorders are reported in DSM-IV-TR. According to DSM-IV-TR, this disorder is more commonly diagnosed in women (75 percent of diagnosed cases are women).

Famlial pattern and genetics: Physical and sexual abuse, neglect, hostile conflict, and early parental loss or separation are more common in childhood histories of patients with this disorder. Borderline personality disorder is five times more common among relatives of probands with this disorder than in the general population. It also increases familial risk for antisocial personality disorder, substance abuse, and mood disorders.

Specific psychopathology $\rightarrow$ weak ego (lack of impulse control and anxiety tolerance)

1) Poor development of self - identity weakness and diffusion

2) Blurred self - object boundaries

3) Partial object relations (All good or all bad)

4) Periodically distorted reality testing

5) Inadequately developed super ego

6) Immature defenses of early phase of development persist into later periods (eg- splitting)

7) Overlap which mood disorder \& PTSD (affective instability)

Comorbidity: These patients are at increased risk for major depression, substance abuse or dependence, eating disorder (notably bulimia), posttraumatic stress disorder (PTSD), and ADHD. Borderline personality disorder co-occurs with most other personality disorders.

Impairment: Impairment is frequent and severe and includes frequent job losses, interrupted education, and broken marriages.

Complications: Complications include psychotic-like symptoms (hallucinations, body image distortions, hypnagogic phenomena, and ideas of reference) in response to stress, premature death or physical handicaps from suicide and suicidal gestures, failed suicide, and self-injurious behavior.

Intervention stratergies: Dialectical behaviour therapy in Borderline PD - The maladaptive behaviours which form part of the borderline syndrome can be viewed as either the product of emotional dysregulation or as attempts by the individual at regulating intense emotional states by maladaptive problem-solving strategies. Dialectical behaviour therapy, as its name suggests, contains within it the notion of opposites; common themes that emerge in therapy with borderline patients, such as acceptance of things as they are (so that there is no need for suicidal action), and change (from former maladaptive types of response) may appear incompatible but are synthesized in the therapy.

The essentials of dialectical behavior therapy are manualized weekly individual psychotherapy, group psychoeducational behavioral skills training, and telephone consultation when considered necessary. Therefore the content comprises a variety of problem-solving techniques including teaching the patient skills to help regulate emotions and tolerate distress, methods for validating the patient's perceptions, and behavioural and psychological versions of meditation skills. 


\section{Cluster C Personality Disorders}

\section{8) AVOIDANT PERSONALITY DISORDER:}

The hallmarks of avoidant personality disorder are pervasive and excessive hypersensitivity to negative evaluation, social inhibition, and feelings of inadequacy

Incidence and prevalence: Prevalence rates of 0.5 to 1.0 percent in the general population and 10 percent for psychiatric outpatients are reported in DSM-IV-TR. According to DSM-IV-TR, this disorder is equally frequent in men and women.

Causes - Specific pychopathology: Increased harm avoidance, behavioural inhibition

Comorbidity: These patients are at increased risk for mood and anxiety disorders (especially social phobia, generalized type). The most common cooccurring disorders are schizotypal, schizoid, paranoid, dependent, and borderline.

Impairment: Impairment can be severe and typically includes occupational and social difficulties.

Courses: Frequently begins in childhood with shyness and fear of strangers and new situations. Disfiguring illness and shyness in childhood predispose children for this personality disorder.

Complications: Complications include social phobia.

Intervention strategies: Psychotherapeutic treatment depends on solidifying an alliance with patients. As trust develops, a therapist must convey an accepting attitude toward the patient's fears, especially the fear of rejection. The therapist eventually encourages a patient to move out into the world to take what are perceived as great risks of humiliation, rejection, and failure. Group therapy may help patients understand how their sensitivity to rejection affects them and others. Assertiveness training is a form of behavior.

\section{9) DEPENDENT PERSONALITY DISORDER:}

The hallmarks of dependent personality disorder are pervasive and excessive need to be taken care of that leads to clinging behavior, submissiveness, fear of separation, and interpersonal dependency.

Incidence and prevalence: This disorder is reported in DSM-IV-TR to be the most frequent of personality disorders. This disorder is equally frequent in men and women (DSM-IV-TR).

Familial pattern and genetics: There is no known familial pattern for this disorder. Chronic physical illness or separation anxiety disorder may predispose for dependent personality disorder.

Causes - Specific pychopathology: Oral stage fixation, increased reward dependence, excess fear of abandonment

Comorbidity: These patients are at increased risk for major depression, anxiety disorders, and adjustment disorder. The most common co-occurring disorders are histrionic, avoidant, and borderline. 
Impairment: Impairment is frequently only mild and typically includes interpersonal relationships and occupational functioning, if independence is required.

Complications: Complications include mood disorders, anxiety disorders, adjustment disorder, and social phobia, as well as low socioeconomic status, poor family, and marital functioning.

\section{0) OBSESSIVE-COMPULSIVE PERSONALITY DISORDER:}

The hallmarks of obsessive-compulsive personality disorder are pervasive and include preoccupation with orderliness, perfectionism, and mental and interpersonal control, at the expense of flexibility, openness, and efficiency.

Incidence and prevalence: Prevalence rates of 1 percent in the general population and 3 to 10 percent for psychiatric outpatients are reported in DSM-IV-TR. According to DSM-IV-TR, this disorder is twice as common in men as in women.

Familial pattern and genetics: Some studies have demonstrated familial aggregation of this disorder.

Causes - Specific pychopathology: Fixation at anal stage, isolation excess use , Low reward dependence, conflict of child's autonomy verses parental wishes $\rightarrow$ shame and criticism $\rightarrow$ OCPD traits

Comorbidity: These patients are at increased risk for major depression and anxiety disorder. There is equivocal evidence for increased risk of OCD.

Impairment: Impairment is frequently severe and typically includes occupational and social difficulties.

Complications: Complications include distress and difficulties when confronted with new situations that require flexibility and compromise and myocardial infarction (secondary to features typical of type A personalities, such as time urgency, hostility, and competitiveness).

Intervention strategies: Obsessive-compulsive personality disorders are often aware of their suffering, and they seek treatment on their own. Over trained and over socialized, these patients value free association and no-directive therapy highly. 
Preventing the completion of their habitual behavior raises patients' anxiety and leaves them susceptible to learning new coping strategies.

Dimensional System of Classifying Personality Disorders[Tyrer, 2000]

\begin{tabular}{|c|c|c|}
\hline $\begin{array}{l}\text { Level of } \\
\text { Severity }\end{array}$ & Description & Definition by Categorical System \\
\hline 0 & No Personality Disorder & $\begin{array}{l}\text { Does not meet actual or subthreshold criteria for any } \\
\text { personality disorder }\end{array}$ \\
\hline 1 & Personality Difficulty & $\begin{array}{l}\text { Meets sub-threshold criteria for one or several } \\
\text { personality disorders }\end{array}$ \\
\hline ? & $\begin{array}{l}\text { Simple-Personality } \\
\text { Disorder }\end{array}$ & $\begin{array}{l}\text { Meets actual criteria for one or more personality } \\
\text { disorders within the same cluster }\end{array}$ \\
\hline 3 & $\begin{array}{l}\text { Complex-(Diffuse) } \\
\text { Personality Disorder }\end{array}$ & $\begin{array}{l}\text { Meets actual criteria for one or more personality } \\
\text { disorders within more than one cluster }\end{array}$ \\
\hline & $\begin{array}{l}\text { Severe-Personality } \\
\text { Disorder }\end{array}$ & $\begin{array}{l}\text { Meets criteria for creation of severe disruption to both } \\
\text { individual and to many in society }\end{array}$ \\
\hline
\end{tabular}

There are several advantages to classifying personality disorder by severity [Murray, 2008]:

- It not only allows for but also takes advantage of the tendency for personality disorders to be comorbid with each other.

- It represents the influence of personality disorder on clinical outcome more satisfactorily than the simple dichotomous system of no personality versus personality disorder.

- This system accommodates the new diagnosis of severe personality disorder, particularly "dangerous and severe personality disorder" (DSPD). Politicians and the public both want to know who comprise the most dangerous group.

\section{EFFECT ON SOCIAL FUNCTIONING}

Social function is affected by many other aspects of mental functioning apart from that of personality. However, whenever there is persistently impaired social functioning in conditions in which it would normally not be expected, the evidence suggests that this is more likely to be created by personality abnormality than by other clinical variables [Nur, Tyrer, Merson, \& Johnson, 2004]. The Personality Assessment Schedule [Tyrer, \& Alexander, 1979].gives social function priority in creating a hierarchy in which the personality disorder creating the greater social dysfunction is given primacy over others in a subsequent description of personality disorder. 


\section{MANAGEMENT \& SPECIFIC APPROACHES}

There are many different forms (modalities) of treatment used for personality disorders [Magnavita, 2004]:

- Individual psychotherapy has been a mainstay of treatment. There are long-term and short-term (brief) forms.

- Family therapy, including couples therapy.

- Group therapy for personality dysfunction is probably the second most used.

- Psychological-education may be used as an addition.

- Self-help groups may provide resources for personality disorders.

- Psychiatric medications for treating symptoms of personality dysfunction or co-occurring conditions.

- Milieu therapy, a kind of group-based residential approach, has a history of use in treating personality disorders, including therapeutic communities.

\section{THERAPEUTIC INTERVENTIONS}

Therapists in this area can become disheartened by lack of initial progress, or by apparent progress that then leads to setbacks. Clients may be experienced as negative, rejecting, demanding, aggressive or manipulative. This has been looked at in terms of both therapist and client; in terms of social skills, coping efforts, defense mechanisms, or deliberate strategies; and in terms of moral judgments or the need to consider underlying motivations for specific behaviors or conflicts. The vulnerabilities of a client, and indeed therapist, may become lost behind actual or apparent strength and resilience. It is commonly stated that there is always a need to maintain appropriate professional personal boundaries, while allowing for emotional expression and therapeutic relationships. However, there can be difficulty acknowledging the different worlds and understandings that client and therapist may live with. A therapist may assume that the kinds of relationships and ways of interacting that make them feel safe and comfortable, have the same effect on clients. As an example at one extreme, people who may in their lives have been used to hostility, deceptiveness, rejection, aggression or abuse, may in some cases be made confused, intimidated or suspicious by presentations of warmth, intimacy or positivity. On the other hand, reassurance, openness and clear communication are usually helpful and needed. It can take several months of sessions, and perhaps several stops and starts, to begin to develop a trusting relationship that can meaningfully address issues [McVey, \& Murphy, 2010].

\section{FUTURE CHALLENGES}

The management and treatment of personality disorders can be a challenging and controversial area, for by definition the difficulties have been enduring and affect multiple areas of functioning. This often involves interpersonal issues, and there can be difficulties in seeking and 
obtaining help from organizations in the first place, as well as with establishing and maintaining a specific therapeutic relationship. On the one hand, an individual may not consider themselves to have a mental health problem, while on the other, community mental health services may view individuals with personality disorders as too complex or difficult, and may directly or indirectly exclude individuals with such diagnoses or associated behaviors [Davison, 2002]. The disruptiveness people with personality disorders can create in an organization makes these, arguably, the most challenging conditions to manage.

\section{CONCLUSION:}

Increased clinical recognition of personality disorder probably reflects its increased presence in the general population. Personality disorder is also a predisposing factor for other psychiatric disorders, possible explanation for the increased interest in personality disorder is its position somewhere in between minor and major psychiatric problems (e.g., adjustment disorder vs. schizophrenia), which makes it interesting to a wide variety of experts and schools, ranging from purely socio dynamic to purely biological. There are numerous possible causes of mental disorders. Genetic dispositions as well as particular life experiences, which may or may not include particular incidents of trauma or abuse. Child abuse and neglect consistently evidence themselves as antecedent risks to the development of personality disorders in adulthood.

\section{REFERENCE:}

"International Statistical Classification of Diseases and Related Health Problems 10th Revision (ICD-10) Version for 2010 (Online Version)".

American Psychiatric Association (2013). Diagnostic and Statistical Manual of Mental Disorders). Arlington, V, A. American Psychiatric Publishing. ISBN 978-0-89042-5558.

Cohen, P., Brown, J., \& Smailes, E. (2001). "Child Abuse and Neglect and the Development of Mental Disorders in the General Population". Development and Psychopathology .PMID 11771917.

Davison, S. E. (2002). "Principles of managing patients with personality disorder". Advances in Psychiatric Treatment.

Magnavita, J. J. (2004). Handbook of personality disorders: theory and practice, John, W., and Sons. ISBN 978-0-471-48234-5.

McVey, D. \& Murphy, N. (2010). Treating Personality Disorder: Creating Robust Services for People with Complex Mental Health Needs. ISBN 0-203-84115-8. 
Murray, R. M. (2008). Psychiatry. Fourth Edition. Cambridge University Press. ISBN 978-0521-60408-6.

Nur, U., Tyrer, P., Merson, S., \& Johnson, T. (2004). "Relationship between clinical symptoms, personality disturbance, and social function: a statistical enquiry". Irish Journal of Psychological Medicine.

Sab, H. (2001). "Personality Disorders," in Smelser, N. J. \& Baltes, P. B., International encyclopedia of the social \& behavioral sciences, Amsterdam. ISBN 978-0-08-043076-8.

Tyrer, P. (2000). Personality Disorders. Diagnosis, Management and Course. Second Edition. London: Arnold Publishers Ltd. ISBN 9780723607366.

Tyrer, P., \& Alexander, J. (1979). "Classification of Personality Disorder". British Journal of Psychiatry. PMID 486849.

WHO (2010) ICD-10: Specific Personality Disorders. 